\title{
EXTENSIONS OF PERIODIC LINEAR GROUPS WITH FINITE UNIPOTENT RADICAL
}

\author{
Richard E. Phillips $\dagger$ \\ Julianne G. Rainbolt ${ }^{1}$ \\ Jonathan I. Hall ${ }^{2}$ \\ Ulrich Meierfrankenfeld ${ }^{3}$ \\ ${ }^{1}$ Department of Mathematics, Saint Louis University, Saint Louis, MO 63103 \\ email: rainbolt@slu.edu \\ ${ }^{2}$ Department of Mathematics, Michigan State University, East Lansing, MI \\ 48824 \\ email: jhall@math.msu.edu \\ ${ }^{3}$ Department of Mathematics, Michigan State University, East Lansing, MI \\ 48824 \\ email: meier@math.msu.edu
}

\section{Dedication}

This article is published posthumously for the author Richard Phillips. The other authors dedicate this paper to his memory.

\begin{abstract}
A group is called $p$-linear if it is isomorphic to a subgroup of $\mathrm{GL}(n, K)$ for some field $K$ of characteristic $p$ and some integer $n$. Let $H$ be a normal subgroup of $G$ and assume that both $H$ and $G / H$ are periodic and $p$-linear. In addition, assume that both $H$ and $G / H$ have finite unipotent radicals and that the HirschPlotkin radical of $H$ is Cernikov. The main result of this article is a proof that under these assumptions $G$ is $p$-linear. An example is provided showing the result is false if the assumption regarding the Hirsch-Plotkin radical is removed.
\end{abstract}




\section{Results}

For finite $n$, the group $G$ is said to be $K$-linear of degree $n$ if it is isomorphic to a subgroup of $G L(n, K)$ and is $p$-linear if it is $K$-linear for some field $K$ of characteristic $p$.

The following theorem was proven as $[1$, Theorem A].

(1.1) TheOREM. Let $G$ be a periodic subgroup of $G L(n, K)$ with trivial unipotent radical. Then for every normal subgroup $H$ of $G$, the image $G / H$ is $K$ linear of degree bounded by a function of $n$.

Here the statement on bounded degree indicates that there is a positive, nondecreasing function $f$ such that the degree of $G / H$ is always less than or equal to $f(n)$.

Wehrfritz [2] gave a second proof of the theorem and was able to weaken the hypotheses by assuming only that $H$ has trivial unipotent radical. A slightly different proof of the theorem is given below (one that in fact includes groups $G$ with finite unipotent radical, as remarked below).

The main concern here is proving a converse result to this theorem. It is not in general the case that an extension of a linear group by a linear group is linear. It is also not clear what shape the best converse to the theorem would take.

Consider the following:

HyPOTHESIS $(*)$

$G$ is a locally finite group and $M$ a normal subgroup of $G$ satisfying:

(i) $M$ is a central product $A Y L_{1} \mathrm{Y} \cdots \mathrm{Y} L_{i} \mathrm{Y} \cdots \mathrm{Y} L_{s}$, for some $s$;

(ii) each $L_{i}$ is a quasisimple group of Lie type in characteristic $p$;

(iii) $A$ is a periodic abelian $p^{\prime}$-group of finite rank $r$;

(iv) $G / M$ is finite.

The rank of a periodic abelian group $B$ is the largest $m$ for which there is a prime $q$ and an elementary abelian $q$-subgroup $C$ of $B$ having order $q^{m}$. If $p=0$, then $s=0$ in (i) and $A$ is periodic abelian of finite rank in (iii).

Each of the groups $L_{i}$ in $(i)$ has, naturally defined, a Lie rank $r_{i}$ and a field of definition $K_{i} \leq \overline{\mathbb{F}}_{p}$. (See [1, Lemma 9(iv)] or Theorem 2.5 below.) (In characteristic $p=0$ we set $\mathbb{F}_{p}=\mathbb{Q}$ hence $\overline{\mathbb{F}}_{p}=\overline{\mathbb{Q}}$.) We define the $M$-rank of $G$ with (*) to be $r+\sum_{i=1}^{s} r_{i}$. We define the field $K^{M}$ to be the smallest subfield of $\overline{\mathbb{F}}_{p}$ that contains each $K_{i}$ and all $q^{k}$ th roots of unity, for every $k$ and all primes $q$ for which $A$ contains a subgroup $\mathbb{Z}_{q^{\infty}}$. Thus $M$ is $K^{M}$-linear.

(1.2) Theorem. If $G$ is a periodic $K$-linear group of degree $n$ with finite unipotent radical $U(G)$, then $G$ has a characteristic subgroup $M$ for which $(G, M)$ satisfies $(*)$, where $p=C h a r(K)$. In this case, $M$ can be chosen so that the $M$-rank of $G$ is bounded by a function of $n$ and the index $|G / M|$ is bounded by functions of $n$ and $|U(G)|$. 
(1.3) Theorem. A group $G$ with $(*)$ is $K^{M}$-linear of degree bounded by a function of its $M$-rank and the index $|G / M|$.

As a corollary, we get Winter's theorem [3]. The usual proofs are more elementary than this; see [4, Theorem 1.L.2] and [5, Theorem 9.5].

(1.4) Corollary. If $G$ is a periodic $K$-linear group with trivial unipotent radical, then $G$ is $\overline{\mathbb{F}}_{p}$-linear, where $p$ is the characteristic of $K$. In particular, $G$ is countable.

Since uncountable unipotent linear groups of exponent $p$ exist, Winter's theorem is false without some restriction on the radical. Nevertheless, because of it we can restrict (most) discussion to the question of whether or not a given group is $p$-linear (that is, linear over some field of characteristic $p$ ).

We have a partial converse to Theorem 1.1.

(1.5) TheOREM. Let $H$ be a normal subgroup of $G$ and assume that

(a) $G / H$ is a periodic p-linear group with finite unipotent radical;

(b) $H$ is a periodic p-linear group with finite unipotent radical;

(c) the Hirsch-Plotkin radical of $H$ is Cernikov.

Then $G$ is p-linear.

A Černikov group is one that satisfies the minimal condition on subgroups and has an abelian subgroup of finite index. (See [4, pp. 28-38]. By [5, Corollary 9.8], in linear groups the second condition is a consequence of the first.) The Hirsch-Plotkin radical of a group is its largest locally nilpotent normal subgroup.

The next section contains proofs of Theorems 1.1-1.5. The final section contains examples. The first example shows that Theorem 1.5 is false under $(a)$ and $(b)$ alone. The other two examples demonstrate the impossibility in Theorem 1.5 of bounding the representing degree of $G$ in terms of $\operatorname{deg} G / H$ and $\operatorname{deg} H$, indeed in terms of the isomorphism class of one of these and the degree of the other.

The problem discussed in this article was one that Richard Phillips was working on during the last years of his life. He wrote an initial draft of this article in conjunction with Julianne Rainbolt. After Richard Phillips' death, Jonathan Hall, Ulrich Meierfrankenfeld, and Julianne Rainbolt completed the revisions of the article. The authors thank Felix Leinen and the referees for helpful remarks on earlier drafts of this article.

\section{Proofs}

Let $G$ be a locally finite group and $\pi$ be any set of primes. Then $O_{\pi}(G)$ denotes the largest normal subgroup of $G$ all of whose elements are $\pi$-elements. If $G$ is periodic and linear in characteristic $p$, then $O_{p}(G)$ is the unipotent radical of $G$. In the following, $E(G)$ denotes the subgroup of $G$ generated by the components of $G$, where a component of $G$ is a subnormal quasisimple subgroup. 
(2.1) Theorem. (Schur, [4, Theorem 1.L.1]) Periodic linear groups are locally finite.

The next two lemmas are elementary.

(2.2) Lemma. If $G$ is $K_{0}$-linear of degree $n$ and $K$ is a subfield of $K_{0}$ such that $\left|K_{0}: K\right|$ is finite, then $G$ is $K$-linear of degree $n\left|K_{0}: K\right|$.

(2.3) Lemma. If $M$ is $K$-linear of degree $m$ and has finite index in $G$, then $G$ is $K$-linear of degree $m|G: M|$ via the induced representation.

(2.4) Lemma. Let $H$ be $K$-linear and $Z$ a finite normal subgroup of the center $Z(H)$. Then $H / Z$ is $K$-linear of degree bounded by a function of the degree of $H$ and $|Z|$.

In particular, if the factors $H_{i}$ are $K$-linear, then a central product $H_{1} Y H_{2}=$ $\left(H_{1} \times H_{2}\right) / Z$ over a finite central subgroup $Z$ is $K$-linear of degree bounded by a function of the degrees of the $H_{i}$ and $|Z|$.

Proof. As the direct product of two $K$-linear groups is $K$-linear with degree equal to the sum of the two degrees, this an immediate consequence of [1, Proposition 3(ii)].

A proof of the following theorem can be found at [6, Lemmas 15.6, 15.10, and Theorem 15.12]. It is very similar to [1, Proposition A] and $[7,1.2]$.

(2.5) Theorem. Let $G$ be a periodic linear group of degree $n$ over a field in characteristic $p$ and having trivial unipotent radical.

Let $\left\{L_{i} \mid 1 \leq i \leq t\right\}$ consist of all components of $G$ that have Lie type in characteristic $p$. The central product $E_{L, p}(G)=L_{1} \mathrm{Y} \cdots \mathrm{Y} L_{t}$ is characteristic in $G$, and $t \leq n / 2$. Furthermore, $G$ has a characteristic abelian subgroup $A$ such that the subgroup $M(G)=A \mathrm{Y} E_{L, p}(G)$ is characteristic in $G$ and has finite index bounded by a function of $n$.

Proof of Theorem 1.2.

$G$ is locally finite by Theorem 2.1 .

As the unipotent radical $U=U(G)$ is finite, $C_{G}(U)$ is a characterisitic subgroup of $G$ of finite index bounded by a function of $|U|$. Therefore we may assume that $U \leq Z(G)$.

Let $\bar{G}=G / U$. Note that $\bar{G}$ is a $K$-linear group of degree $n$ with trivial unipotent radical.

Let $\bar{N}=M(\bar{G})$ be the subgroup $\bar{A} Y \bar{L}_{1} Y \cdots Y \bar{L}_{s}$ of Theorem 2.5. Let $L_{i}$ be the derived group of the preimage of $\bar{L}_{i}$ in $G, A$ the $p^{\prime}$-part of the preimage of $\bar{A}$, and $M=A \mathrm{Y} L_{1} \mathrm{Y} \cdots \mathrm{Y} L_{s}$. Then $M$ is characteristic in $G$, and the pair $(G, M)$ satisfies $(*)$.

The various $\operatorname{rank}\left(L_{i}\right)$ are bounded by a function of $n$ [1, Lemma 9(b)(i)]. Since $A$ is $p^{\prime}$ and periodic, it has finite rank bounded by a function of $n$ by Maschke's Theorem. 


\section{Proof of TheOrem 1.3.}

By $(i v)$ and Lemma 2.3, we may assume $G=M$. Each $L_{i}$ is $K^{M}$-linear by assumption and has a center that is finite of order bounded by a function of the Lie rank of $L_{i}$ ([1, Lemma 10(viii)] or Lemma 2.7). Thus $E(G)=L_{1} \mathrm{Y} \ldots \mathrm{Y} L_{s}$ is $K^{M}$-linear by Lemma 2.4 and has finite center of order bounded in terms of the Lie ranks of the $L_{i}$. By definition, $\left(K^{M}\right)^{\times}$contains a copy of $\mathbb{Z}_{q \infty}$ whenever $A$ has infinite $q$-part, so $A$ is $K^{M}$-linear of degree equal to its rank $r$. A second application of Lemma 2.4 then proves that $G=M=A$ Y $E(G)$ is $K^{M}$-linear, as desired. Furthermore, its degree is controlled as described.

Proof of Corollary 1.4.

As $G$ is periodic and $K$-linear, it has $(*)$ by Theorem 1.2 and so is $\overline{\mathbb{F}}_{p}$-linear by Theorem 1.3.

\section{Proof of TheOREM 1.1 .}

By Theorem 1.2, $G$ has $(*)$ for a normal subgroup $M$, and the $M$-rank of $G$ and $|G / M|$ are both bounded by functions of $n$. In $\bar{G}=G / H$, set $\bar{M}=$ $M H / H$. Then the pair $(\bar{G}, \bar{M})$ inherits $(*)$, so by Theorem $1.3 \bar{G}$ is linear of degree bounded in terms of the $\bar{M}$-rank of $\bar{G}$ (at most the $M$-rank of $G$ ) and $|\bar{G}: \bar{M}|(\leq|G: M|)$. Thus the degree of $G / H$ is bounded by a function of $n$, as desired.

REMARK. The same proof actually gives something slightly stronger than Theorem 1.1. We need only require that the unipotent radical of $G$ be finite, in which case the representation degree of $G / H$ is bounded by a function of $n$ and of the order of the radical.

(2.6) Lemma. Let $B$ be a class 2 nilpotent periodic group with $B^{\prime} \leq H \leq$ $Z(B)$. Assume that $B / H$ has finite rank and that $H$ is Cernikov. Then $B$ has an abelian subgroup of finite index and finite rank.

Proof. The central subgroup $H$ is Cernikov and so has only finitely many prime divisors; let the set of these be $\pi$. The set of $\pi^{\prime}$-elements of $B$ then forms a characteristic abelian subgroup $O_{\pi^{\prime}}(B) \simeq O_{\pi^{\prime}}(B / H)$ of finite rank. Let $B=O_{\pi^{\prime}}(B) \times B_{0}$, where $B_{0}=O_{\pi}(B)$ consists of all $\pi$-elements of $B . B_{0} / H$ is of finite rank and is, in fact, Cernikov since $\pi$ is finite. But then $B_{0}$ is Cernikov by [4, Lemma 1.E.7]. In particular it contains an abelian subgroup $A_{0}$ of finite rank and of finite index in $B_{0}$. The subgroup $O_{\pi^{\prime}}(B) \times A_{0}$ is then abelian of finite rank and has finite index in $B$, as desired.

(2.7) Lemma. Let periodic $N$ have a central subgroup $H$ such that $\bar{N}=N / H$ is a central product $\bar{M}_{1} \mathrm{Y} \ldots \mathrm{Y} \bar{M}_{t}$ of finitely many infinite quasisimple groups $\bar{M}_{i}$ of Lie type. Then $N=H \mathrm{Y} E(N)$, where $E(N)=N_{1} \mathrm{Y} \cdots \mathrm{Y} N_{t}$ has finite center and is a central product of quasisimple groups $N_{i}$ of Lie type with $\bar{N}_{i}=\bar{M}_{i}$. 
Proof. By the Three Subgroups Lemma, $Z_{2}(N)=Z(N)$. Also $N^{\prime}=$ $\left[N^{\prime} H, N^{\prime} H\right] \leq N^{\prime \prime}$; so $N^{\prime}$ is perfect, and $N=H$ Y $N^{\prime}$. Indeed $N / H=$ $N^{\prime} H / H \simeq N^{\prime} / N^{\prime} \cap H$ has image $N^{\prime} / Z\left(N^{\prime}\right)$. Therefore $N^{\prime}=E(N)$, the central product of the components $N_{i}=\left(M_{i}\right)^{\prime}$, the derived subgroups of the preimages $M_{i}$ of the various $\bar{M}_{i}$.

Each simple periodic infinite Lie type group $L$ is a direct limit of finite simple groups of the same Lie type, and so any element of its multiplier occurs already within the multiplier of some finite subgroup of Lie type. Exceptional multipliers for finite Lie type groups occur only over small fields, and the canonicial multipliers come from the natural or spin representations of fixed degree bounded in terms of the Lie rank [8]. Thus the multiplier of $L$ is finite of order bounded by the rank of $L$ and comes from a representation of degree also bounded by the rank. (See also [1, Lemma 10].)

We next prove a special case of Theorem 1.5.

(2.8) TheOREM. Let $H$ be a normal subgroup of $G$ and assume that

(a) $G / H$ is a periodic p-linear group with finite unipotent radical;

(b) $H$ is finite.

Then $G$ is p-linear with finite unipotent radical.

Proof. By Theorem $2.1 \mathrm{G} / \mathrm{H}$ is locally finite, so $G$ itself is locally finite (by [4, Lemma 1.A.2]).

Since the quotient of any linear group by its unipotent radical is also linear, we may replace $H$ by the preimage in $G$ of the unipotent radical of $G / H$. Thus we may assume that $G / H$ has trivial unipotent radical.

As $H$ is finite, $C_{G}(H)$ has finite index in $G$. Replacing $H$ by $H \cap C_{G}(H)$ and $G$ by $C_{G}(H)$, we may by Lemma 2.3 assume that $H \leq Z(G)$.

By Theorem 1.2, $M(Q)$ has finite index in $Q$, and so its preimage in $G$ has finite index. Thus by Lemma 2.3 in proving $G$ to be $p$-linear we can assume $Q=M(Q)=A \mathrm{Y} E(Q)=A \mathrm{Y} L_{1} \mathrm{Y} \cdots \mathrm{Y} L_{s}$. Indeed again by Lemma 2.3 we just need to show that the preimage $G_{0}$ of $A Y E_{\infty}(Q)$ is $p$-linear, where $E_{\infty}(Q)=L_{1} \mathrm{Y} \cdots \mathrm{Y} L_{t}$ only includes those components $L_{i}$ of $Q$ that are infinite.

By Lemma $2.7, G_{0}=B Y E\left(G_{0}\right)$, where $E\left(G_{0}\right)$ is a central product of Lie type groups and $B(\geq H)$ has class at most 2 with $B / H=A$. By Lemma 2.6, $B$ has an abelian subgroup $B_{0}$ of finite index and finite rank. As $O_{p}(G) \leq H$ is finite, $B_{0}$ in turn has a finite index abelian $p^{\prime}$-subgroup $A_{0}$ of finite rank. Therefore $G_{0} \geq A_{0} Y E\left(G_{0}\right)$ is $p$-linear by Theorem 1.3, as desired.

\section{Proof of TheOrem 1.5.}

By Theorem 2.1 both $H$ and $G / H$ are locally finite, so $G$ itself is locally finite (by [4, Lemma 1.A.2]). By Theorem 2.8 we may assume that $O_{p}(H)$, the unipotent radical of $H$, is trivial.

By Theorem 2.5 $M(H)$ is characteristic of finite index in $H$, so by Theorem 2.8 we may assume $H=M(H)=C$ Y $E(H)$, where $C(\geq Z(E(H)))$ is abelian, 
characteristic in $H$, and Cernikov. By [1, Lemma 9] $C_{G}(E(H)) E(H)$ has finite index in $G$, so we may take $G=C_{G}(E(H)) E(H)$ (by Lemma 2.3 again). By [4, Theorem 1.F.3], any periodic subgroup of $\operatorname{Aut}(C)$ is finite, so we may further assume $G=M(H) C_{G}(M(H))$. Thus $G=C_{G}(M(H))$ Y $C$ Y $E(H)$, a central product (indeed $C \leq C_{G}(M(H))$ ). $E(H)$ has finite center by [1, Lemma 10] (or Lemma 2.7). As $E(H)(\leq H)$ is $p$-linear by hypothesis, when we have proven that $C_{G}(M(H))$ Y $C=C_{G}(M(H))$ is $p$-linear we will be done by Lemma 2.4. Linear $G / H=M(H) . C_{G}(M(H)) / H \simeq C_{G}(M(H)) / H \cap C_{G}(M(H))=$ $C_{G}(M(H)) / C$, so we are reduced to the case $C=H \leq Z(G)$.

As $O_{p}(H)=1, O_{p}(G)$ is isomorphic to the unipotent radical of $Q=G / H$ and is, in particular, finite. Thus by Theorem 2.8 we may assume that $O_{p}(G)$ and $O_{p}(Q)$ are trivial. We now complete the proof as in the last two paragraphs of the proof of Theorem 2.8.

\section{Examples}

(3.1) Lemma. There is an infinite sequence of primes

$$
q_{1}<p_{1}<\cdots<q_{i}<p_{i}<\cdots
$$

for $i \in \mathbb{Z}^{+}$, with $q_{i} \mid p_{i}-1$.

Proof. The proof is by induction on $i$. Let $q_{1}$ be any prime number. A theorem of Dirichlet $\left[9\right.$, p. 250] asserts that the sequence $\left\{1+k q_{1} \mid k=\right.$ $1,2, \ldots\}$ contains an infinite number of primes. Let $p_{1}$ be any such prime. Then $q_{1} \mid\left(p_{1}-1\right)$. Now let $q_{2}$ be a prime greater than $p_{1}$.

Suppose that we have chosen $q_{1}, \ldots, q_{s+1}$ and $p_{1}, \ldots, p_{s}$ such that

$$
q_{1}<p_{1}<\cdots<q_{s}<p_{s}<q_{s+1}
$$

Now choose the prime $p_{s+1}$ in the sequence $\left\{1+k q_{s+1} \mid k=1,2, \ldots\right\}$ and the prime $q_{s+2}$ with $q_{s+2}>p_{s+1}$.

\section{(3.2) EXAmPle.}

We first show that Theorem 1.5 is false when only $(a)$ and $(b)$ are assumed (even when $(a)$ and $(b)$ are strengthened to require that the unipotent radicals be trivial).

For $p_{i}, q_{i}\left(i \in \mathbb{Z}^{+}\right)$as in Lemma 3.1, let $S_{i}$ be cyclic of order $p_{i}$ and $R_{i}$ cyclic of order $q_{i}$. Let $F_{i}=S_{i} . R_{i}$ be the Frobenius group of order $p_{i} q_{i}$. Then $F^{k}=\oplus \sum_{i=1}^{k} F_{i}$ has representation degree at least $2 k$ in each characteristic, even though it is the extension $S^{k} \cdot R^{k}$ of cyclic $S^{k}=\oplus \sum_{i=1}^{k} S_{i}$ of degree 1 over any prime not in the sequence (and degree at most 2 in general) by cyclic $R^{k}=\oplus \sum_{i=1}^{k} R_{i}$ also of degree 1 over any prime not in the sequence (and degree at most 2 in general).

Thus $F^{\infty}=\oplus \sum_{i} F_{i}=\lim _{k} F^{k}$ is not linear in any characteristic even though it is the extension of rank 1 linear $S^{\infty}=\oplus \sum_{i} S_{i}=\lim _{k} S^{k}$ by rank 1 
linear $R^{\infty}=\oplus \sum_{i} R_{i}=\lim _{k} R^{k}$. The Hirsch-Plotkin radical of $F^{\infty}$ is $S^{\infty}$ and is not C̆ernikov.

\section{(3.3) ExAmple.}

The groups $F^{k}$ of the first example show that the degree of linear $G$ can not be bounded in terms of the degree, $\operatorname{deg} H$, of the normal subgroup $H$ and $\operatorname{deg} G / H$. We can refine the example to show that knowledge of $\operatorname{deg} H$ and the isomorphism type of $G / H$ is still not enough in general to bound the degree of linear $G$.

Indeed, let linear $F^{(k)}=F^{k} \oplus \sum_{i>k} R_{i}$ be the split extension of $S^{k}$ (as before) by $R^{\infty}$ (with the $R_{i}$, for $i>k$, acting trivially). As $F^{k} \leq F^{(k)}$, the representation degree still goes to infinity, but now $F^{(k)} / S^{k} \simeq R^{\infty}$, for all $k$.

\section{(3.4) EXAMPLE.}

Finally, as a complement to the previous example, we show that the degree of linear $G$ can not in general be bounded in terms of $\operatorname{deg} G / H$ and the isomorphism type of $H$.

Fix a characteristic $p$ and a prime $q \neq p$. Let, for $n \in \mathbb{Z}^{+}$,

$$
E(n)=\left\langle x, y, z \mid x^{q^{n}}=y^{q^{n}}=z^{q^{n}}=1,[x, y]=z,[x, z]=[y, z]=1\right\rangle .
$$

Let $E^{*}(n)$ be the central product of $E(n)$ and $\mathbb{Z}_{q^{\infty}}$ with $z$ identified with an element of order $q^{n}$ of $\mathbb{Z}_{q^{\infty}}$. Then $H=\mathbb{Z}_{q^{\infty}}$ is $p$-linear of degree 1 and $E^{*}(n) / H \simeq Z_{q^{n}} \times Z_{q^{n}}$ is $p$-linear of degree 2 .

We claim that the degree of $E(n)$ (and hence of $E^{*}(n)$ ) in characteristic $p$ is $q^{n}$. Abelian $W=\langle x, z\rangle$ has index $q^{n}$, so this is an upper bound on the degree. Consider the restriction of a faithful representation to $W$. There is some degree 1 constituent $\zeta$ with $W=\langle w, z\rangle=C_{E(n)}(w)$, where $\langle w\rangle=\operatorname{ker}(\zeta)$. Then by

Clifford's Theorem the $\zeta^{y^{i}}$, for $i=0, \ldots, q^{n}-1$, are distinct constituents of the restriction.

\section{References}

[1] Phillips, R.E.; Rainbolt J.G. Images of Periodic Linear Groups. Arch. Math. 1998, 71, 97-106.

[2] Wehrfritz, B.A.F. Periodic Normal Subgroups of Linear Groups. Arch. Math. 1998, 71, 169-172.

[3] Winter, D.J. Representations of Locally Finite Groups. Bull. Amer. Math. Soc. 1968, 74, 145-148.

[4] Kegel, O.H.; Wehrfritz, B.A.F. Locally Finite Groups; North Holland: Amsterdam, 1973.

[5] Wehrfritz, B.A.F. Infinite Linear Groups; Springer-Verlag: BerlinHeidelberg-New York, 1973. 
[6] Meierfrankenfeld, U. Lecture Notes on Locally Finite Groups, 1997 (available from http://www.math.msu.edu/ meier/)

[7] Wehrfritz, B.A.F. Locally Finite Normal Subgroups of Absolutely Irreducible Skew Linear Groups. J. London Math. Soc. 1985, 32 (2), 88-102.

[8] Griess, R.L. Jr. Schur Multipliers of the Known Finite Simple Groups II. In Finite Groups; Proc. Sympos. Pure Math. 1980, 37, 279-282.

[9] Ribenboim, P. The Book of Prime Number Records; Springer-Verlag: Berlin-Heidelberg-New York, 1988. 